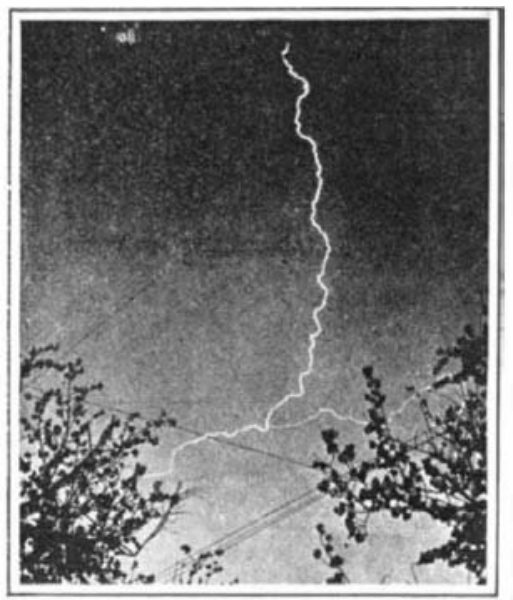

\title{
New Ideas About Lightning
}

A Postscript to the Schoolbooks and Encyclopædias

By C. Fitzhugh 'Talman

C LEMENTARY text-books still generally follow E Arago in classifying the visible electrical dis charges of the atmosphere under three distinctive headings: zigzag lightning, sheet lightning, and ball lightning.

The term zigzag lightning is a misnomer, and like other misnomother misnom-
ers tends to perpetuate a mistaken idea. In a n c i en: paintings and sculptures the lightnings grasped in the grasped in the hand of Jove or clutched in the talons of
his eagle bristle with sharp angles. Exactly the same

kind of lightning is still represented by artists. Arago and his contemporaries firmly believed in angular lightning. Its existence was first denied by James Nasmyth, in 1856, and his contention that so-called zig-zag lightning is really sinuous was soon after confirmed by photography. Nasmyth's drawings illustrating the contrast between the lightning of art and the lightning of nature are reproduced in Fig. 1 The need of reiterating his announcement at this late date is shown by the fact that lightning flashes still zig-zag their way through the drawings of many still zig-zag their way through the drawings of many
contemporary illustrators of story books. It is not so long ago that they were definitely banished from the school books.

The camera has solved many problems concerning the forms of lightning, but has also raised a number of new ones. The use of a moving camera, which ture at succes ture at successive moments of time, introduced by Weber and Hoffert in 1889, and further applied by Walter in 1901 and Larsen in 1902 revolutionized ou knowledge of this sulject. This method has been fur ther improved by Walter during the past two years. He now uses two cameras, installed side by side, one o which is fixed, while the other is turned slowly on vertical axis by clockwork. Two photographs are thu all the flashes occurring within a certain field within a certain field,
while the other analyzes while the other analyzes the flashes that have a sensible duration; and pictures shows the rela tions in time of the various phases of the phenomenon. (See Fig. 2.)

Briefly stated, tho camera has given us the following information in regard to lightning:

Visible lightning is due to the incandescence of the air along the path of an electric dischargewhich may be sinuous, spiral or looped (disregarding the sheet and globular forms for the present) and is usually acrompanied by numerous branches. The flash as a rule consists of several sparks (i. e., separate discharges) following the same path. Each spark is generally instantaneous, in the ordinary sense of the term (sometimes lasting less than one five-thousandth second, according to Schmidt), but the intervals be 1B.Walter, "Ueber Doppelaufnahmen von Blitzen," Jahrb. Hamb. Wiss.
Anstalten, 27, 1909. 5 Beiheft. Hamburg, 1910.

tween the sparks may average one tenth second, so that the total duration of the flash is often half a second or more. These successive discharges along the same path give lightning its flickering appearance. The electric discharge does not, as a rule, immediately bridge over the whole space between cloud and
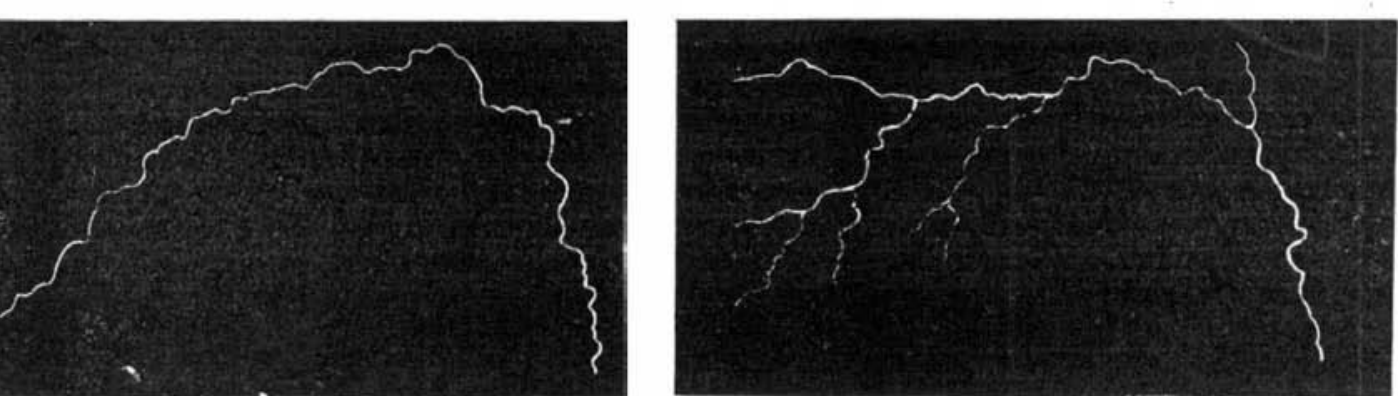

Natural Lightning

Fig. 1. -Nasmyth's drawings of lightning, real and unreal. Quarterly Journal of the Royal Meterrolosical Society

earth or cloud and cloud. It builds up its path grad ually; the electric current feels its way, so to speak The first feeble discharges extend only part way along the ultimate path, and end as brush discharges in the air. Then comes a sudden and powerful discharge along the whole path. This may completely equalize the potential between the two bodies connected by the flash; or, after a brief pause, a series of discharges arabe some the remarkable photo graphs taken by Walter, at Hamburg, show these three classes of phenomena very clearly; and they have been named by this investigator the "prelim inary," "primary," and "after" discharges, respective1y. ("Vorentladungen," "Anfangsentladung," "Nachentladungen.") The time occupied by the preliminary discharges has been found to vary between 0.001 and 0.02 second. The after-discharges may continue for half a second, or more, as stated above.

In a photograph of a lightning flash made with a camera moved across the direction of the flash so as to reveal its successive phases (see Fig. $2 b$ ), the successive sparks are usually easy to distinguish, but are not separated by intervals of complete darkness. The whole band has a more or less blurred effect, apparently indicating that the lllumination persisted from one spark to the next. Often this glow is stronger at

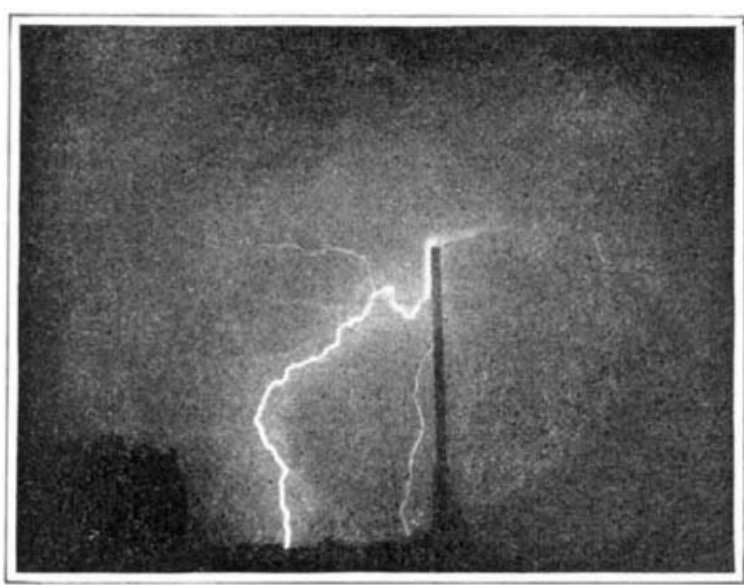

Fig. $2 a$

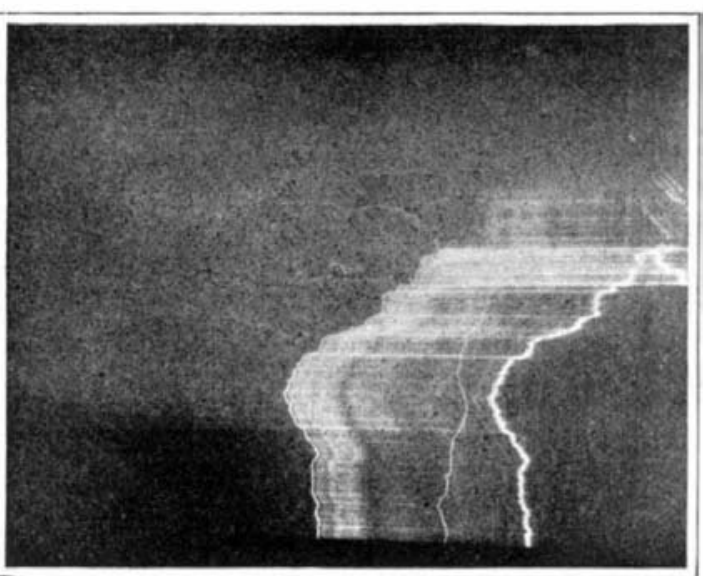

Fig. 20

armly advocated by W. J. S. Lockyer, among others." Those who accept this view call the phenomenon an "afterglow." According to K. E. F. Sch'midt ${ }^{3}$, the glow is due to phosphorescence. An analogous phenomenon een in Geissler tubes containing air has recently been phosphoresdue to the due to the
reaction of $\mathrm{ni}$. tric oxide a nd ozone formed in the discharge. According to Walter the afterglow has no objective existence. He claims that the horiseen in the moving-camera photographs are chiefly due to the superposition of those portions of the path of the discharge that run parallel with the motion of the camera. Lastly, we must suppose that the diffuse glows seen about arl the brighter features of a lightning photograph are at least partly the result of internal reflections in the camera and other defects in the technique of the photographic process.

"Ribbon" lightning is common in lightning photographs; $i$ e the photograph very often shows a band, rather than a line of light; but this is, in most cases, simply due to an accidental or intentional movement of the camera transversely to the direction of the flash, or sometimes to the same inherent defects of the camera that account in part for the afterglow. Walter finds that with a perfectly stationary camera the apparent breadth of an average stream of lightning would, if real, indicate that the path was over three feet in diameter. In these cases he believes that the breadth of the path is lllusory; the imperfect definition of the objective for bright objects having spread the image as much as tenfold. The same effect is seen in astronomical photographs; the brighter stars appearing on the negative not as points, but as broad disks. The human retina is subject to the same defect; and we may thus account for the frequently reported co cribon" lightning, as een with the naked eye. In some cases a flash of lightning, as recorded by a stationary camera, or as seen by the eye, may be more or less broadened owing to a lateral shifting by the wind, of the air channel along which the suecer along which the successive discharges occur; but that there should be anything like a uniform shifting all along the path of a flash of average lengthi. e., several thousand feet-is, in the nature of things, extremely unlike1y. The wind effect might ly. The wind effect might cause an irregular thick-

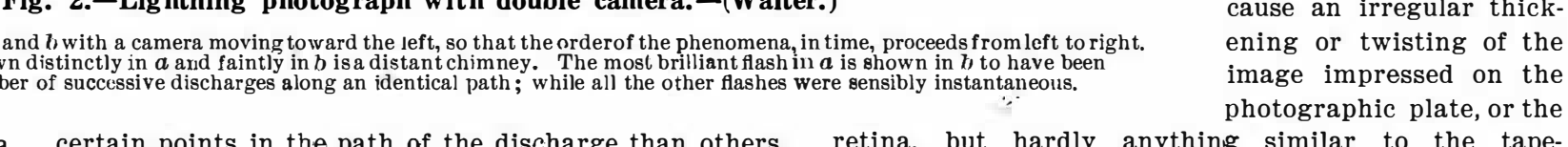

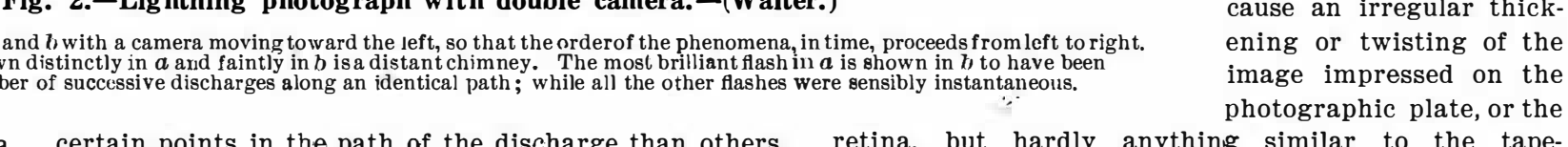

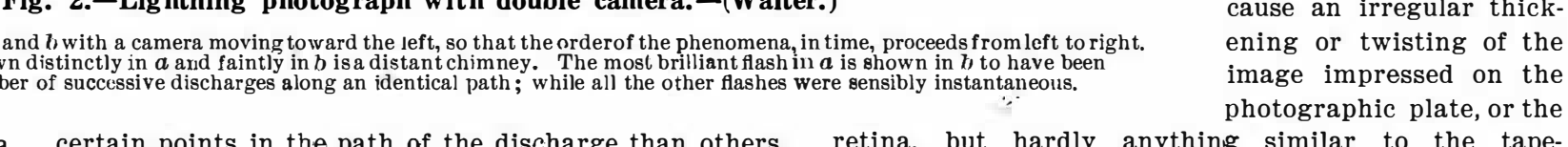

certain points in the path of the discharge than others, so that the moving camera gives the appearance of horizontal striation to the broadened image of the flash. The true nature of this glow has not yet been set tled beyond dispute. The most obvious explanation is that the incandescence of the air persists for a time after the discharge is passed. This explanation was offered by Touchet some years ago, and has been retina, but hardly anything similar to the tapelike stream of light ination of many hundred photographs of lightination of many hundred photographs of lightby Kayser, at Berlin, July 16th, 1884-in which some-

'Comptes rendus, 140, 1905, p. 1031 
thing approaching the ribbon form was registered by an absolutely motionless camera ${ }^{4}$; and even in this case the "ribbon" is so irregular in width as to hardly deserve this name. I think, moreover, it is safe to assume that the flash photographed by Kayser was rather near the camera, so that the portion of it shown in his picture had not a great absolute length "Beaded" lightning-also known as "chapleted" or "pearl" lightning-has several times been drawn but

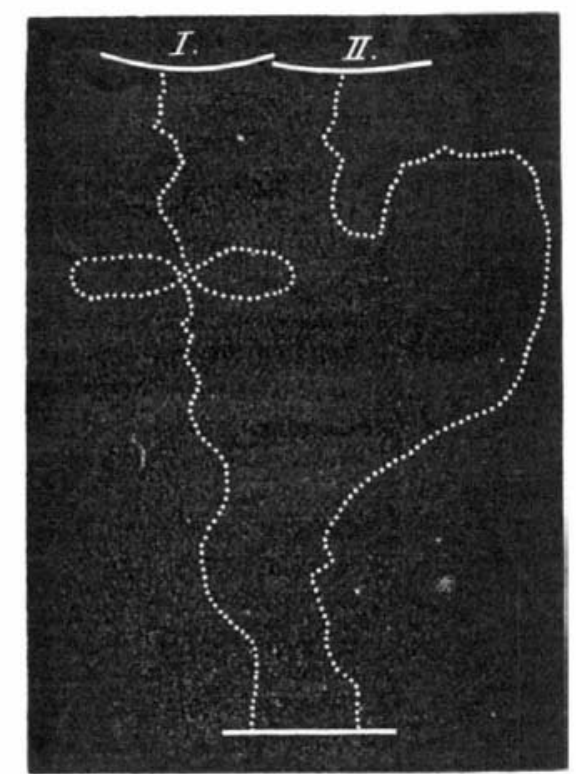

Fig. 3.-Drawing of two flashes of "beaded" lightning. (After Endemann, Metesrologische Zeitschrift, 1888.)

very rarely photographed. It is described as consisting of a series of bright points giving more or less the effect of a string of pearls. (See Fig. 3.) In several cases an ordinary linear flash is said to have resolved itself into this beaded form before fading out of sight. Lockyer $^{3}$ thinks thạt in all cases it is simply a discontinuous afterglow; though, as stated above, the existence of the afterglow has been denied. Photo graphs with a moving camera appear to show that the afterglow is strongest at the points along a light ning flash where the direction of the flash changes Thls is illustrated in Fig. 4. It will be seen that the This is illustrated in Fig. 4. It will be seen that the yer, the trails impressed upon the moving photographic plate by the afterglow, are most pronounced at the bends and loops in the path of the discharge. It is evident that if these bright spots persisted long enough after the original flash to be perceptible to the naked eye, they would produce exactly the effect deBcribed as beaded lightning. Unfortunately, this phenomenon has been rarely observed, and we are not able to say positively whether the "beads" sometimes able to say positively whether the "beads" sometimes
appear without a previous continuous flash, in which case we should have to seek another explanation. It may be that in some cases what is seen is simply several portions of a continuous flash visible through interstices in the clouds. "Stellar" lightning (often seen in connection with volcanic eruptıons) is explained by Prinz as probably an effect of perspective. Suppose the branching flash shown in Fig. 6 to be seen by an observer at either $A$ or $B$, i. e., in the line of the flash. It will present the appearance shown in Fig. 5

"Black" lightning-a common feature of lightning photographs, generally in connection with ordinary bright flashes-is often explained as a reversai due to over-exposure. (See Scientific A Merican, 1910, p. 463.) This explanation hardly seems to account for This explanation hardly seems to account for
the fact that the small, and presumably relatively faint, lateral branches of a bright flash are so often of this character. Since the discovery by A. W. Clayden, in 1889 of what is now known as the "Clayden effect," it has been generally believed that after relatively faint flashes have been impressed on the plate, a subsequent general illumination of the field by light reflected from clouds causes these flashes to print dark, or under some conditions to be en. tirely obliterated. ${ }^{6}$

"Sheet" lightning is the name applied to diffuse; and usually noiseless discharges. Common usage extends this term to include diffuse reflections of ordinary linear dis. charges, which are themselves hidden by clouds or below the horizon; especially the "heat" lightning that plays along the horizon on summer evenings, and is merely the reflection of a thunder

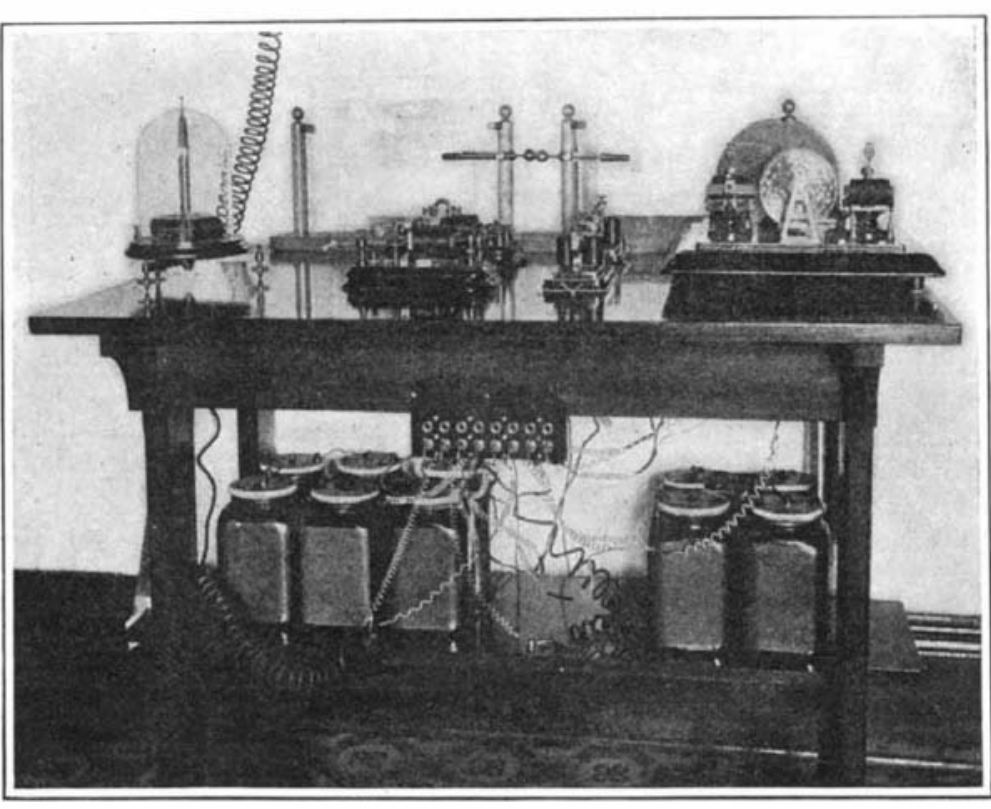

Fig. 7. -Odenbach's ceraunograph as installed at St. Ignatius College Observatory, Cleveland, Ohio.

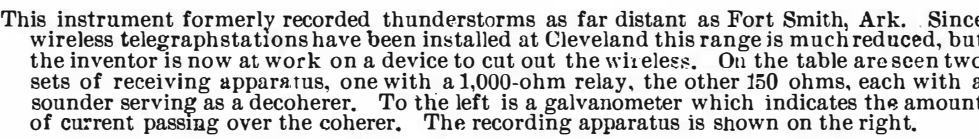
negative electricity.

According to this hypothesis the heavy rain of a thunderstorm should be positively charged, and that such is the case was shown by extensive observations made by Simpson in India. However, within the highly electrified cloud there must be a rapid combination of the negatively charged water drops, which would fall as a more gentle rain; and this assumption is

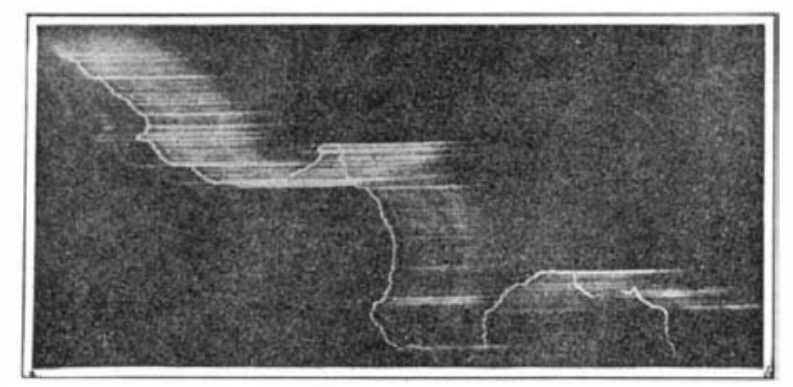

Fig. 4. - Lockyer's explanation of “beaded " lightning. (Photograph by Walter.)

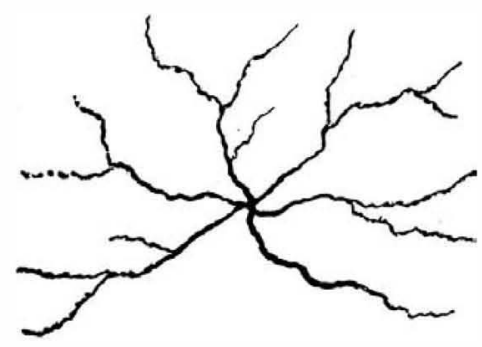

Fig. 5._-"Stellar" lightning.

(After Jrinz.) ittle over two years old, and is due to a brilliant young English physicist, George Simpson, attached to the

Simpson's hypothesis is based upon the fact, well attested by laboratory experiments, that the breaking up of drops of water involves a separation of positive from negative electricity; in other words, a production of both positive and negative ions. In this process a greater number positively charged; if e, they retain the latter being set free in the air. About three times as many negative as positive ions are thus released. Now a thunderstorm is accompanied by a strong upward movement of the air; so strong that small drops cannot fall through it, while large drops, which would be heavy enough to fall through such a vertical current if they could retain their integrity, are broken up by the air and then carried aloft, where they tend to accumulate, recombine and fall again. This process may be repeated over and over again, so that the and at the same time negative ions are being set free, which are carried by the ascending air current to the upper part of the cloud, where they unite with the cloud particles and give them a strong negative charge. Thus a continuous separation of electricity is in prog. ress. Ultimately the positively charged drops accumu-

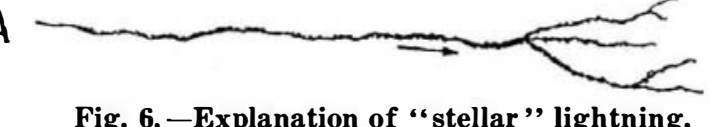

Fig. 6. -Explanation of "stellar" lightning. B (After Prinz.)

also confirmed by observation. Simpson's hypothesis may be said to have supplanted that of Wilson and Gerdien, according to which the separation of the positive from the negative ions was due to the fact that the latter more readily become the nuclei of aqueous condensation. It was supposed that, in an ascending air current, the dust was sifted out by condensation upon it in the lower strata; as the air, thus freed of its dust, continued to rise, it was cooled until it had reached such a degree of supersaturation that condensation could occur upon the negative ions, which would thus be sifted out in their turn. The objections raised to this hypothesis are chiefly quantitative rather than qualitative, and it is still an open question whether it may or may not account in part for the potential gradients observed in the atmosphere.

\section{Lightning Recorders.}

Even a hasty survey of recent progress in the study of lightning should take note of the ingenious use of the methods of wireless telegraphy in observing and recording electrical discharges in the atmosphere.

A flash of lightning sets up electromagnetic waves in the ether. These may be received, at great distances from their source, by a suitable antenna, and sent through a coherer (using this term in the generic sense), which thus becomes conducting to a galvanic current; the latter actuates a relay, which closes a secondary circuit, and brings into play the recording apparatus, which is usually a pen tracing a record on a revolving drum. The forms of lightning recorder that thus make a continuous registration on a sheet of paper are called generically ceraunographs (a term introduced by Odenbach in 1891). Several forms of the ceraunograph have been devised by Boggio-Lera, Fenyi, Schreiber, Lancetta, Odenbach, Garcia Mollà and others. Their radius of action is several hundred miles. By the use of several relays Boggio-Lera and Turpain have produced instruments that record not only the occurrence, but also approximately the dis tance, of an electrical discharge. There are also thunderstorm recorders that make an audible record, by ringing a bell or otherwise. In the ingenious instrument invented by Tommasina-known as the electro-radio-

late to such an extent, or they reach a place where the updraft is so weakened that they fall as positively ${ }^{7}$ Metenrolnoisahe Zeitschrift. 17, 1900, pp. 448-457; Mnnthly - Proc. Roy. Soc., ser. A, 82, 1909, p. 169, ffg. phone or ceraunophone-the observer uses a telephone receiver, whereby he is enabled to listen to a thunder storm far beyond the range of the unaided ear, and to gain some idea of the movement of the storm by noting the variations in the intensity of the sound. 